\title{
Soluciones analíticas y numéricas de esfuerzos mecánicos en placas rectangulares isotrópicas
}

Juan P. Cardona ${ }^{1 *}$, John J. Leal' ${ }^{2}$, José U. Castellanos ${ }^{1}$ y José E. Ustariz ${ }^{1}$

(1) Universidad Cooperativa de Colombia, Facultad de Ingeniería, Av. Caracas №. 37-15, Bogotá D.C.-Colombia. (correo-e: juan.cardonag@campusucc.edu.co; jose.castellanos@campusucc.edu.co; jose.ustariz@campusucc.edu.co)

(2) Universidad Nacional de Colombia, Facultad de Ingeniería y Administración, Kr. $32 \mathrm{~N}^{\circ} 12-00$, Palmira, Valle del Cauca. (correo-e: jlealgom@unal.edu.co)

* Autor a quien debe ser dirigida la correspondencia.

Recibido Abr. 8, 2021; Aceptado Jun. 7, 2021; Versión final Ago. 19, 2021, Publicado Dic. 2021

\begin{abstract}
Resumen
En este estudio se desarrolla y valida un modelo para simular numéricamente los esfuerzos normales y cortantes inducidos sobre las caras superior e inferior de una placa plana rectangular e isotrópica. La placa está apoyada en sus cuatro bordes y la presión es uniformemente distribuida sobre toda su área. Las soluciones analíticas se hallaron por medio de la ecuación general de placas utilizando el método series dobles de Fourier. Las soluciones numéricas se obtuvieron por el método de elementos finitos (software ANSYS 19.1). La validación del modelo se realiza comparando estadísticamente las soluciones analíticas y numéricas de los esfuerzos inducidos en un experimento clásico con tres niveles de presión. Los resultados muestran que no hay diferencia estadística significativa entre los dos tipos de soluciones. Se concluye que el modelo de simulación numérica es válido.
\end{abstract}

Palabras clave: soluciones analíticas; modelo; simulación; esfuerzos mecánicos; elementos finitos; placas planas

\section{Analytical and numerical solutions for mechanical stresses on isotropic rectangular plates}

\begin{abstract}
In this study, a model is developed and validated to numerically simulate normal and shear stresses exerted on the upper and lower faces of a rectangular and isotropic flat plate. The plate is supported on its four edges and the pressure is evenly distributed over its entire area. The general equation of plates using the double Fourier series method is applied to find analytical solutions. Numerical solutions are obtained by the finite element method (ANSYS 19.1 software). The model is validated statistically by comparing analytical and numerical solutions in a classic experiment that considered three pressure levels. The results show that there is no statistical significant difference between the two types of solutions. Therefore, it is concluded that the numerical simulation model is valid.
\end{abstract}




\section{INTRODUCCIÓN}

Actualmente las soluciones a problemas de diseño en ingeniería se dan desde tres enfoques: analítico, experimental y numérico. El primero de ellos utiliza soluciones basadas en modelos matemáticos de leyes o principios físicos, si el diseño se simplifica mucho o las condiciones de operación del mismo son muy idealizadas los resultados pueden presentar desviaciones significativas a las del producto real, lo que puede ocasionar distintos tipos de problemas en el diseño final. Por otra parte, en el enfoque experimental se construyen prototipos físicos a escala reducida o real del sistema que se quiere diseñar, que serán sometidos a condiciones reales de operación, y así los resultados obtenidos con este tipo de enfoque son muy precisos, ya que representan fielmente las características físicas y constructivas del sistema, además, en estos ensayos generalmente se utilizan dispositivos e instrumentos para la medición de datos que garantizan una comprensión detallada de los fenómenos de interés. El desarrollo de este tipo de pruebas experimentales requiere una mayor inversión respecto al enfoque analítico, tanto en costos de construcción del prototipo, como en la contratación de personal especializado y en la infraestructura necesaria para los ensayos.

Tanto en el enfoque analítico, como en el enfoque numérico de sistemas físicos, se consideran hipótesis simplificadoras en relación con la realidad, se relacionan variables que utilizan leyes físicas que resultan en ecuaciones diferenciales ordinarias o parciales, se establecen condiciones iniciales o de operación, y se obtienen soluciones aproximadas al comportamiento del prototipo real. Sin embargo, el enfoque numérico presenta una serie de características destacadas, que la hacen una solución extremadamente versátil y eficiente para el desarrollo de proyectos de ingeniería. Por ejemplo, la facilidad para parametrizar los modelos numéricos, posibilita una mejor comprensión del funcionamiento del sistema, la evaluación de diferentes condiciones de diseño y el desarrollo de una configuración más óptima, que responda todos los criterios de diseño y seguridad, este enfoque, requiere una inversión razonablemente superior al método analítico (debido a la infraestructura de hardware, licencias de software y entrenamiento de personal), pero tiene un costo significativamente menor a un ensayo experimental.

Una de las técnicas computacionales actuales más utilizadas para resolver éste tipo de ecuaciones es el método de elementos finitos (MEF), una poderosa herramienta que resuelve los modelos matemáticos con mucha precisión, considera los materiales, las geometrías de los diseños y las condiciones iniciales de operación, permite la utilización de diversas técnicas y métodos de solución; generalmente las soluciones se realizan en tres pasos, el preprocesamiento, procesamiento y posprocesamiento, etapas en las cuales se consideran distintos factores para ser tenidos en cuenta y obtener resultados satisfactorios a la hora de las simulaciones. Actualmente esta técnica se ha utilizado en una amplia variedad de problemas, y tiene distintos enfoques para sus soluciones. (Croninl 2001; Moreno et al., 2007; Vanam et al., 2012)

Roylance (2001), Pallares y Rodríguez (2009), Vélez et al. (2009), exponen la importancia del uso de herramientas de simulación como el programa comercial de elementos finitos ANSYS como alternativa viable para la solución de problemas de ingeniería, ya que constituye un ejercicio de interiorización teórica y práctica de fenómenos naturales o procesos que de otra manera sería complejo explicar desde el punto de vista cuantitativo y aun cualitativo. Un software de elementos finitos como ANSYS contienen herramientas que permiten el modelado y la simulación de un gran número de fenómenos, estructuras y materiales, para lo cual divide su operación en etapas o módulos, donde generalmente se definen aspectos tales como geometría del modelo, tipos de elementos en los que se dividirá, asignación de propiedades de los materiales y generación de la malla para definir los nodos y uniones entre elementos. (Gurunath, 2015; Alfonso et al., 2015; Ramírez y Rodan, 2016; Muhammad et al., 2020).

El presente artículo muestra el desarrollo y validación de un modelo de simulación numérica que utiliza elementos finitos con el software ANSYS 19.1. para determinar el nivel de esfuerzo normal y cortante que soportan las caras de una placa plana rectangular homogénea e isotrópica, simplemente apoyada en sus cuatro bordes, sometida a una presión uniformemente distribuida. El modelo se desarrolló en el marco de una Investigación de corte cuantitativo que utilizó un diseño experimental clásico con tres niveles de carga, bajo, medio y alto. La validación comparó estadísticamente las soluciones analíticas y numéricas, mediante una prueba t. Las dimensiones de la placa se tomaron con fines de simulación, y al comparar los resultados analíticos y numéricos, se concluye que el modelo de simulación es válido y por tanto se pueden ajustar los parámetros correspondientes, como dimensiones geométricas, materiales y cargas.

\section{PLACAS PLANAS}

Las placas planas como componentes estructurales básicos se utilizan ampliamente en áreas de la ingeniería, como la aeroespacial (aviones y misiles), civil (estructuras arquitectónicas, puentes, pavimentos), marina (barcos, contenedores) y mecánica (estructuras hidráulicas, instrumentos y piezas de máquina), entre otras. Las placas son miembros estructurales delimitados por dos planos paralelos, llamados caras, y una superficie 
llamada borde o límite. La distancia entre las caras planas se llama espesor el cual es pequeño en comparación con otras dimensiones características de la placa como longitud y ancho y se mide en una dirección normal al plano medio, para fines de cálculos técnicos como los presentados en este artículo se define una placa plana delgada como aquella en la cual la relación de su espesor a la longitud de su dimensión más pequeña debe ser inferior a 1/20 (Manjunath et al., 2014; Yuwaraj y Param, 2016; Hassan, 2019).

En el análisis de una placa plana es común dividir su espesor en mitades iguales por un plano paralelo a las caras, este plano se llama plano medio de la placa. En muchos casos, las placas están expuestas a cargas externas que producen deformaciones, esfuerzos y se pueden producir fallas mecánicas, por tanto, es necesario realizar diseños adecuados de las estructuras que utilizan éste tipo de placas planas, para determinar con precisión aspectos como: resistencia a esfuerzos, uso eficiente, características de comportamiento bajo cargas, deformación o pandeo de la placa. (Ventsel y Krauthammer, 2001; Sadrnejad et al., 2009; Mohtaram et al., 2012).

De acuerdo con Ugural (2010), excepto para tipos de geometrías y cargas simples, las soluciones analíticas de la ecuación 1 se producen solo con considerable dificultad, debido a ello es común intentar una solución por el método inverso, el cual se basa en soluciones supuestas para la deflexión de la placa plana (w) que satisfacen la ecuación gobernante y las condiciones de contorno. Desde este punto de vista el método más poderoso de este tipo es la serie de Fourier, donde, una vez se ha encontrado una solución para carga sinusoidal, se puede manejar cualquier otra carga por series infinitas. Este enfoque ofrece como importante ventaja el hecho de que una sola expresión puede aplicarse a toda la superficie de la placa plana.

\section{Modelo matemático para la deflexión en placas planas}

El modelo matemático que describe el comportamiento de las placas planas, ha sido ampliamente estudiado por distintos autores Timoskenko y Gere (2009), Herrera (2011) y Birman (2011) y se representa por medio de la ecuación 1, conocida como la ecuación general de placas, que permite hallar las soluciones analíticas para la deflexión $\mathrm{w}$ de una placa plana con módulo de rigidez $\mathrm{D}$ cuando está sometida a una presión uniformemente distribuida $\mathrm{p}$ :

$$
\frac{\partial^{4} w}{\partial x^{4}}+2 \frac{\partial^{4} w}{\partial x^{2} \partial y^{2}}+\frac{\partial^{4} w}{\partial y^{4}}=\frac{p}{D}
$$

Para el caso particular de una placa rectangular simplemente apoyada en sus cuatro bordes, de espesor $t, y$ dimensiones a y b, con módulo de elasticidad $\mathrm{E}$, coeficiente de Poisson $\vartheta$, a la cual se le aplica una presión uniformemente distribuida con valor $P_{0}$, se pueden calcular los momentos flectores $M_{x}$ y $M_{y}$ y el momento torsor $\mathrm{M}_{\mathrm{xy}}$, para lo cual se utilizan las series de Fourier, ecuaciones (2), (3) y (4). Es importante notar que los momentos flectores $M_{x}$ y $M_{y}$ son ambos cero en $(x=0, x=a)$ y $(y=0, y=b)$, respectivamente. Sin embargo, el momento de torsión $M_{x y}$ no desaparece en los bordes ni en las esquinas de la placa. (Ventsel y Krauthammer,2001; Timoschenko y Gere,2009; Ugural, 2010)

$$
\begin{aligned}
& M_{x}=\frac{16 p_{0}}{\pi^{4}} \sum_{m=1,3,5 \ldots n=1,3,5 . .}^{\infty} \sum_{m n\left[(m / a)^{2}+(n / b)^{2}\right]^{2}}^{\infty} \operatorname{sen} \frac{m \pi x}{a} \operatorname{sen} \frac{n \pi y}{b} \\
& M_{y}=\frac{16 p_{0}}{\pi^{4}} \sum_{m=1,3,5 \ldots n=1,3,5 . .}^{\infty} \sum_{n n\left[(m / a)^{2}+(n / b)^{2}\right]^{2}}^{\infty} \frac{\vartheta(m / a)^{2}+(n / b)^{2}}{a} \operatorname{sen} \frac{n \pi y}{b} \\
& M_{x y}=\frac{16 p_{0}(1-\vartheta)}{\pi^{4} a b} \sum_{m=1,3,5 \ldots n=1,3,5 . .}^{\infty} \sum_{m n\left[(m / a)^{2}+(n / b)^{2}\right]^{2}}^{\infty} \cos \frac{m \pi x}{a} \cos \frac{n \pi y}{b}
\end{aligned}
$$

\section{Solución analítica de esfuerzos mecánicos}

Los esfuerzos mecánicos normales $\sigma_{x}$ y $\sigma_{y}$ y cortantes $\tau_{x y}$ se inducen en una placa plana rectangular, sometida a cargas transversales a lo largo de los ejes $\mathrm{x} y$ y respectivamente y cortantes en el plano $\mathrm{xy}$, los cuales varían de un punto a otro de la placa y además dependen de la deflexión de la misma (w). Dichos esfuerzos están dados por las ecuaciones (5), (6) y (7). (Timoskenko y Gere, 2009; Ugural, 2010). 


$$
\begin{aligned}
& \sigma_{x}=-\frac{E z}{1-\vartheta^{2}}\left(\frac{\partial^{2} w}{\partial x^{2}}+\vartheta \frac{\partial^{2} w}{\partial y^{2}}\right) \\
& \sigma_{y}=-\frac{E z}{1-\vartheta^{2}}\left(\frac{\partial^{2} w}{\partial y^{2}}+\vartheta \frac{\partial^{2} w}{\partial y^{2}}\right) \\
& T_{x y}=\frac{E z}{1+\vartheta} \frac{\partial^{2} w}{\partial x \partial y}
\end{aligned}
$$

Los valores de esfuerzos normales $\left(\sigma_{x}, \sigma_{y}\right)$, y cortantes $\left(\tau_{x y}\right)$ soportados en las superficies de las caras superior e inferior de la placa en función de los momentos flectores $M_{x}$ y $M_{y}$, los torsores $M_{x y}$; están dados por las ecuaciones (8), (9) y (10). (Ventsel y Krauthammer,2001; Timoskenko y Gere, 2009; Ugural,2010). Los esfuerzos mecánicos se miden en $\mathrm{Pa}$, los momentos por unidad de longitud en $\mathrm{N}$ y las fuerzas por unidad de longitud tienen unidades $\mathrm{N} / \mathrm{m}$.

$$
\begin{aligned}
& \sigma_{x}= \pm \frac{6 M_{x}}{t^{2}} \\
& \sigma_{y}= \pm \frac{6 M_{y}}{t^{2}} \\
& \tau_{x y}= \pm \frac{6 M_{x y}}{t^{2}}
\end{aligned}
$$

\section{MATERIALES Y MÉTODOS}

La placa plana rectangular que se consideró para el siguiente estudio es de acero estructural que tiene las dimensiones siguientes, largo $b=64 \mathrm{~m}$, ancho $a=32 \mathrm{~m}$ y espesor $\mathrm{t}=0,02 \mathrm{~m}$, sus cuatro bordes están simplemente apoyados y se encuentra sometida a una presión uniformemente distribuida $\mathrm{P}_{0}=1000 \mathrm{~Pa}$ tal como se observa en la figura 1.

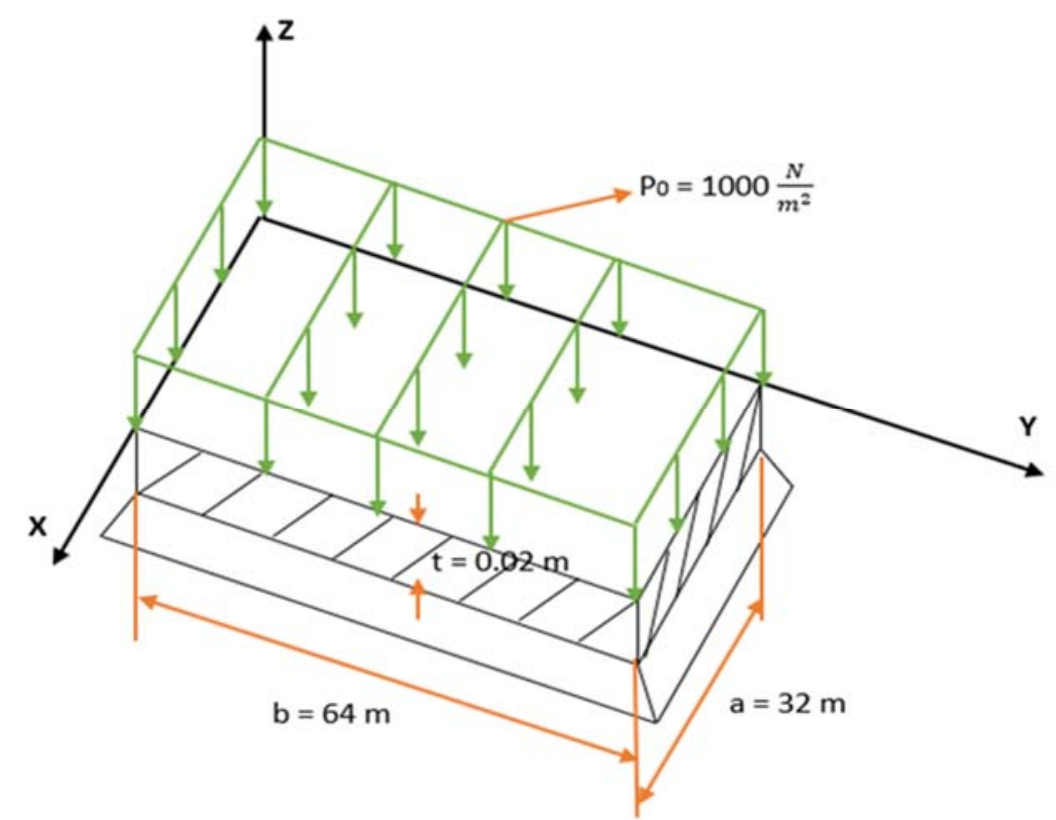

Fig. 1: Placa rectangular simplemente apoyada en sus cuatro bordes sometida a presión uniformente distribuida

La investigación que se realizo fue de tipo analítico con enfoque cuantitativo, el experimento consideró la presión uniformente distribuida que actúa sobre la placa rectangular plana como variable independiente (variable de entrada) y los niveles de esfuerzo normales y cortantes como variable dependiente (variable de salida), se utilizó un diseño experimental clásico con tres niveles de presión, bajo: 10 Pa, medio: 100 Pa y alto:1000 Pa y se obtuvieron los niveles de esfuerzo correspondientes a dichos niveles de presión. Las soluciones numéricas obtenidas en la simulación se utilizó el software ANSYS 19.1 se consideraron como los datos experimentales y las soluciones analíticas serán los datos de control. 
La investigación fue de tipo analítico con enfoque cuantitativo, el experimento consideró la presión uniformente distribuida que actúa sobre la placa rectangular plana como variable independiente (variable de entrada) y los niveles de esfuerzo normales y cortantes como variable dependiente (variable de salida), se utilizó un diseño experimental clásico con tres niveles de presión, bajo: $10 \mathrm{~Pa}$, medio: $100 \mathrm{~Pa}$ y alto:1000 Pa y se obtuvieron los niveles de esfuerzo correspondientes a dichos niveles de presión. Las soluciones numéricas obtenidas con el software ANSYS 19.1 fueron tomadas como los datos experimentales y las soluciones analíticas serán los datos de control.

El modelo de simulación numérica desarrollado fue resuelto mediante el método de elementos finitos con el software ANSYS 19.1 que permite visualizar las soluciones. En el módulo Engenieering Data del software se escogió el acero estructural como material de la placa plana, igualmente se establecierón sus propiedades de elasticidad, isotropía, y homogeneidad. Las propiedades utilizadas en el modelo fueron: módulo de elasticidad

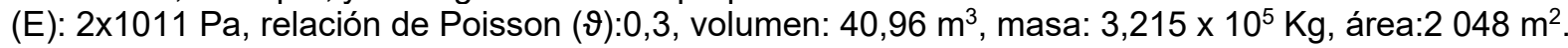

La geometría de la placa plana se modeló en el módulo DesingModeler®, se utilizó una longitud b de $64 \mathrm{~m}$, un ancho de $32 \mathrm{~m}$, un área superficial de $2048 \mathrm{~m}^{2}$, consta de una cara, cuatro ejes y cuatro vértices. La placa es considerada como plana, de espesor cero, sus características se muestran en la figura 2 . El mallado de la geometría se desarrolló en el módulo Mechanical®, la malla se estableció mediante la herramienta mesh, la placa de acero se discretizó con elementos bidimensionales rectangulares tipo lámina identificados como QUAD-4(Shell) que se adaptan mejor a la geometría en estudio.

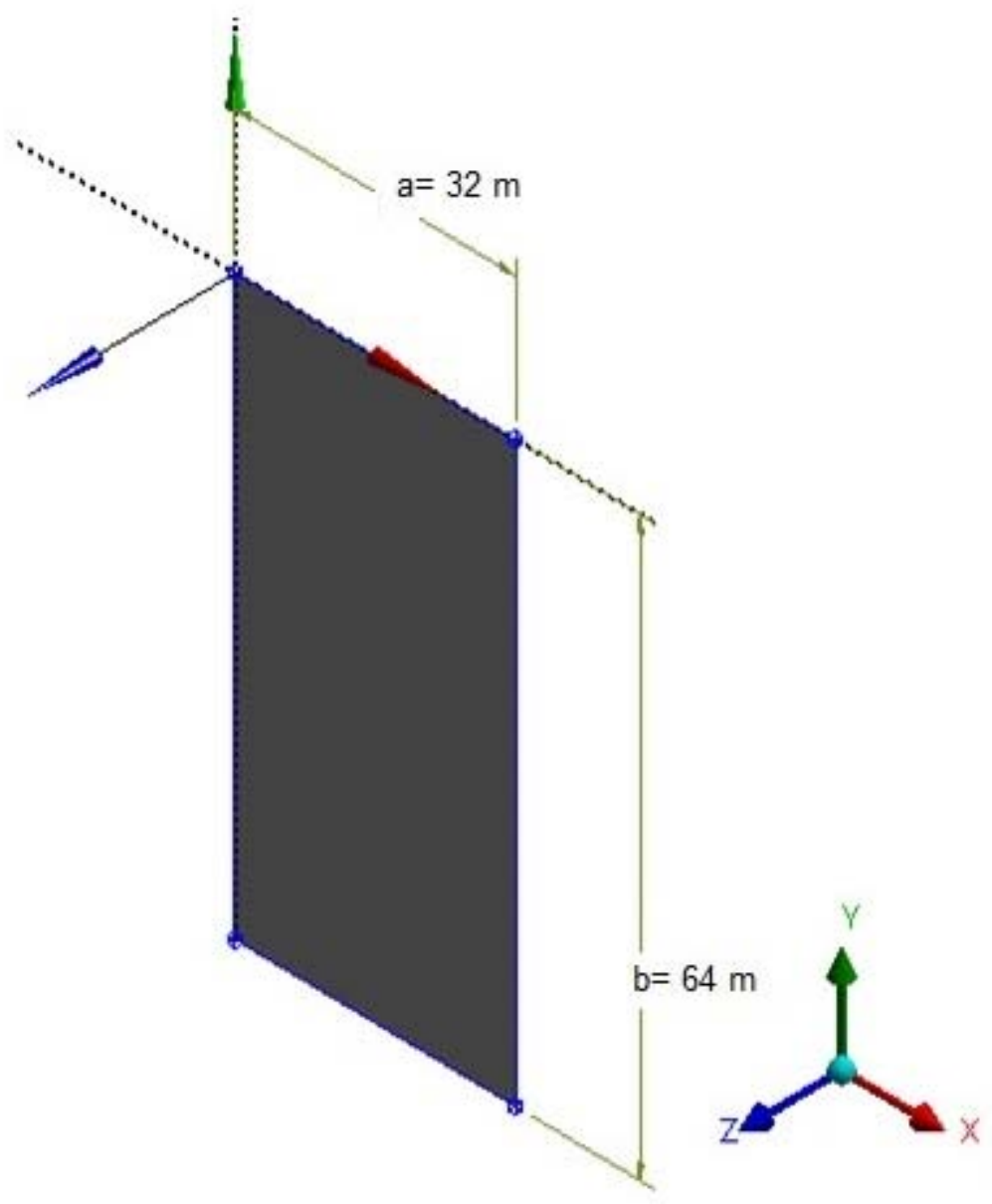

Fig. 2: Geometría de la placa plana rectangular generada en ANSYS

Los QUAD-4 (shell) tienen cuatro nodos y seis grados de libertad; tres traslaciones y tres rotaciones. La malla obtenida consta de 2145 nodos y 2048 elementos cada uno de tamaño un metro, tal como se muestra en la figura 3. Con respecto de la métrica de la malla se resaltan los siguientes valores: Calidad de la malla (Element Quality) en promedio 0,99; relación de aspecto (Aspect Ratio) en promedio 1,01; ortogonalidad (Orthogonal Quality) en promedio 0,99 y oblicuidad (Skewness) en promedio 1,0242e-002. En el módulo ANSYS ${ }^{8}$ StaticStructural ${ }^{\circledR}$, se configuraron los dominios, se establecieron las condiciones de borde simplemente apoyadas para la placa plana y se aplicó una presión uniformente distribuida en la superficie de la placa con un valor de $1000 \mathrm{~Pa}$ tal como se muestra en la figura 4. 


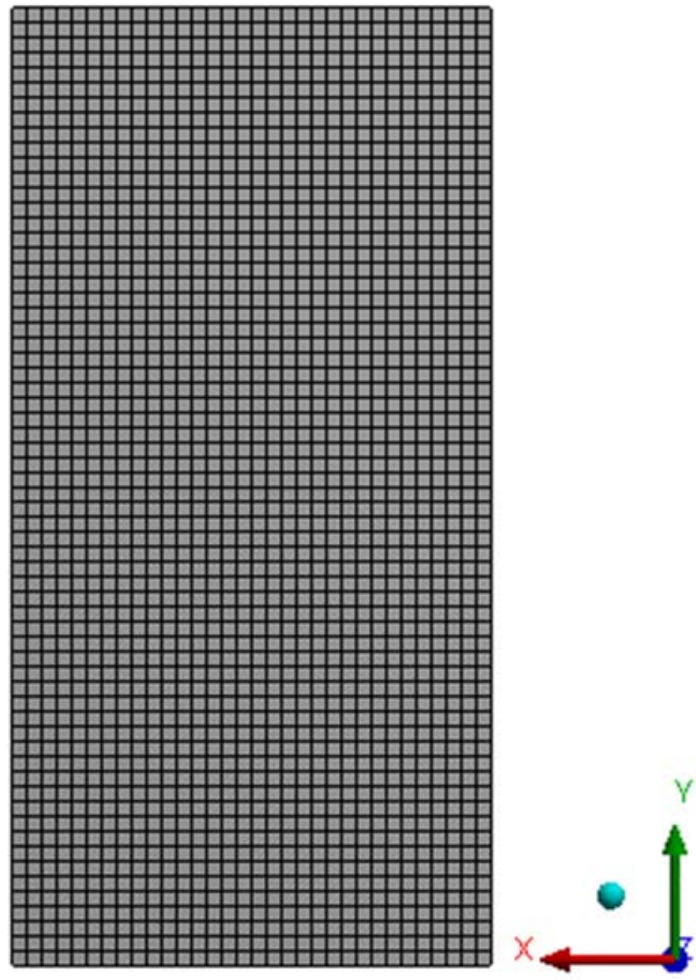

Fig. 3: Mallado de la placa plana rectangular generada en ANSYS

A: Static Structural

Pressure: $1000, \mathrm{~Pa}$

Simply Supported:0, $\mathrm{m}$

Simply Supported 2:0,m

D Simply Supported 3:0,m

Simply Supported 4:0,m
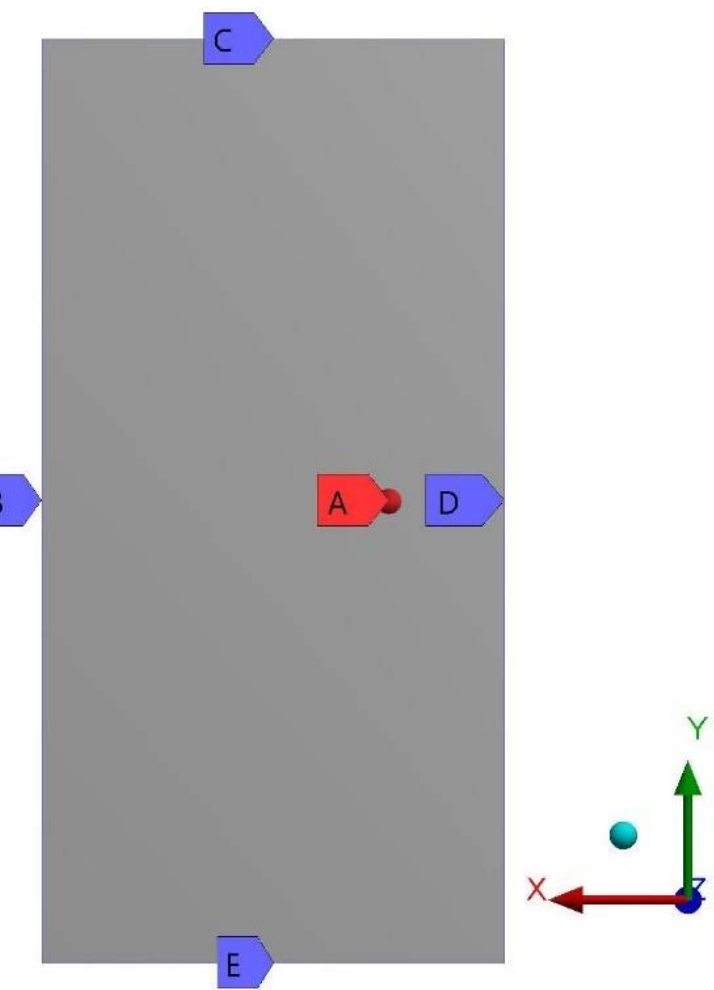

Fig. 4: Carga y condiciones de borde de la placa plana rectangular generada en ANSYS

\section{RESULTADOS Y DISCUSIÓN}

El estudio de los valores de esfuerzo normal $\left(\sigma_{x}, \sigma_{y}\right)$ y cortante $\left(\tau_{x y}\right)$ en las caras superior e inferior de la placa rectangular plana se realizó para tres niveles de presión a saber, bajo $10 \mathrm{~Pa}$, medio $100 \mathrm{~Pa}$ y alto $1000 \mathrm{~Pa}$. A continuación, se presentan los resultados obtenidos para el nivel más alto: inicialmente se muestran las soluciones analíticas halladas mediante la utilización de las series dobles de Fourier, ecuaciones (8), (9) y (10); posteriormente se presentan los resultados obtenidos mediante la visualización de la simulación numérica realizada mediante el software ANSYS 19.1. 


\section{Resultados analíticos}

Los valores de esfuerzo normal a lo largo del eje $\mathrm{x}\left(\sigma_{\mathrm{x}}\right)$ se calcularon por medio de la ecuación 8 para diferentes puntos a lo largo de la línea central de la placa cada b/16 y los resultados hallados se muestran en la tabla 1.

Tabla 1: Soluciones analíticas del esfuerzo normal $\left(\sigma_{\mathrm{x}}\right)$ en diferentes puntos a lo largo de a/2 para presión de 1000 Pa.

\begin{tabular}{|c|c|c|c|c|c|c|c|c|}
\hline$L$ & $\mathrm{~b} / 16$ & $\mathrm{~b} / 8$ & $3 b / 16$ & $\mathrm{~b} / 4$ & $5 b / 16$ & $3 \mathrm{~b} / 8$ & $7 \mathrm{~b} / 16$ & $\mathrm{~b} / 2$ \\
\hline $\begin{array}{c}\sigma_{x} \\
{[\mathrm{MPa}]}\end{array}$ & $4,1647 \cdot 10^{2}$ & $\begin{array}{c}7,6324 \\
\cdot 10^{2}\end{array}$ & $1,0422 \cdot 10^{3}$ & $1,2350 \cdot 10^{3}$ & $1,3870 \cdot 10^{3}$ & $1,4912 \cdot 10^{3}$ & $1,5365 \cdot 10^{3}$ & $1,5453 \cdot 10^{3}$ \\
\hline $\mathrm{L}$ & $9 b / 16$ & $5 \mathrm{~b} / 8$ & $11 \mathrm{~b} / 16$ & $3 \mathrm{~b} / 4$ & $13 \mathrm{~b} / 16$ & $7 \mathrm{~b} / 8$ & $15 \mathrm{~b} / 16$ & \\
\hline $\begin{array}{c}\sigma_{\mathrm{x}} \\
{[\mathrm{MPa}]}\end{array}$ & $1,5365 \cdot 10^{3}$ & $1,4912 \cdot 10^{3}$ & $1,3870 \cdot 10^{3}$ & $1,235 \cdot 10^{3}$ & $1,0346 \cdot 10^{3}$ & $7,6324 \cdot 10^{3}$ & $4,1647 \cdot 10^{3}$ & \\
\hline
\end{tabular}

La figura 5 muestra los resultados de la convergencia del valor de esfuerzo normal a lo largo del eje $x\left(\sigma_{x}\right)$ en la superficie de la placa y en la mitad de la misma (a/2, b/2) al aplicar una presión de $1000 \mathrm{~Pa}$, dichas soluciones se obtuvieron mediante la aplicación de la ecuación 8. La convergencia de la solución está dada en función del número de armónicos de la serie de Fourier.

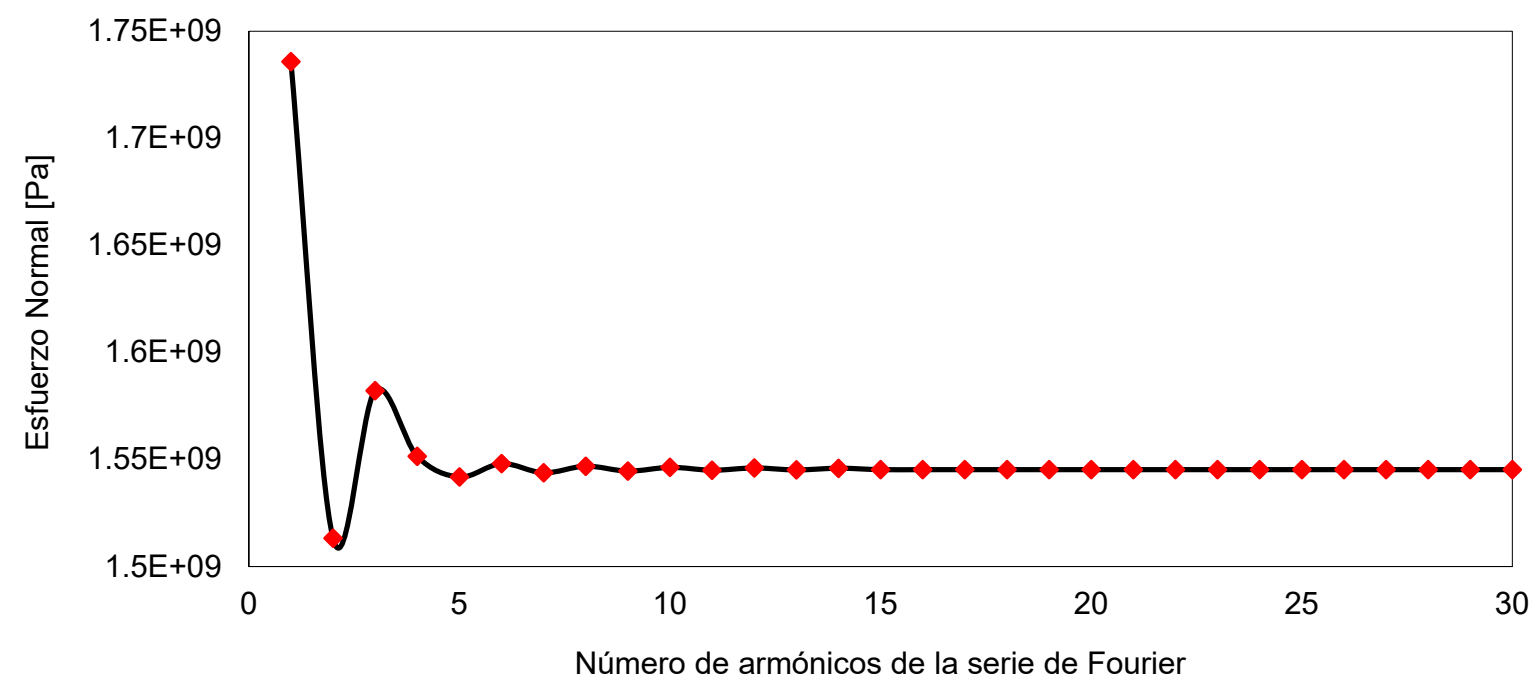

Fig. 5: Convergencia del esfuerzo normal a lo largo del eje $x\left(\sigma_{x}\right)$ en el centro de la placa con el número de armónicos

La tabla 2 muestra los resultados de las soluciones analíticas del valor del esfuerzo cortante $\left(\tau_{\times y}\right)$ en la superficie de la placa y en la mitad de la misma $(a / 2, b / 2)$, dichas soluciones se obtuvieron mediante la aplicación de la ecuación 10, fundamentada en el uso de series de Fourier. La convergencia de la solución en función del número de términos de la serie de Fourier se muestra en la figura 6 . El procedimiento anterior se aplicó para diferentes puntos a lo largo de la línea central de la placa cada b/16 y los resultados hallados se muestran en la tabla 3.

Tabla 2: Soluciones analíticas del esfuerzo cortante $\tau_{\text {xy }}$ en diferentes puntos a lo largo de a/2.

\begin{tabular}{|c|c|c|c|c|c|c|c|}
\hline L & $b / 16$ & $\mathrm{~b} / 8$ & $3 b / 16$ & $\mathrm{~b} / 4$ & $5 b / 16$ & $3 b / 8$ & $7 b / 16$ \\
\hline $\begin{array}{c}\tau_{\mathrm{xy}} \\
{[\mathrm{Pa}]}\end{array}$ & $-2,8903 \cdot 10^{5}$ & $-1,7203 \cdot 10^{6}$ & $-6,544 \cdot 10^{5}$ & $-5,2824 \cdot 10^{5}$ & $-3,7708 \cdot 10^{5}$ & $-2,4656 \cdot 10^{5}$ & $-1,9877 \cdot 10^{5}$ \\
\hline L & $\mathrm{b} / 2$ & $9 b / 16$ & $5 \mathrm{~b} / 8$ & $11 b / 16$ & $3 b / 4$ & $13 b / 16$ & $7 \mathrm{~b} / 8$ \\
\hline $\begin{array}{c}\tau_{x y} \\
{[\mathrm{~Pa}]}\end{array}$ & $-9,6465 \cdot 10^{2}$ & $-1,9877 \cdot 10^{5}$ & $-2,4656 \cdot 10^{5}$ & $-3,7708 \cdot 10^{5}$ & $-5,2824 \cdot 10^{5}$ & $-6,5446 \cdot 10^{5}$ & $-1,7203 \cdot 10^{6}$ \\
\hline L & $15 b / 16$ & & & & & & \\
\hline $\begin{array}{c}\tau_{x y} \\
{[\mathrm{~Pa}]}\end{array}$ & $-2,8903 \cdot 10^{5}$ & & & & & & \\
\hline
\end{tabular}




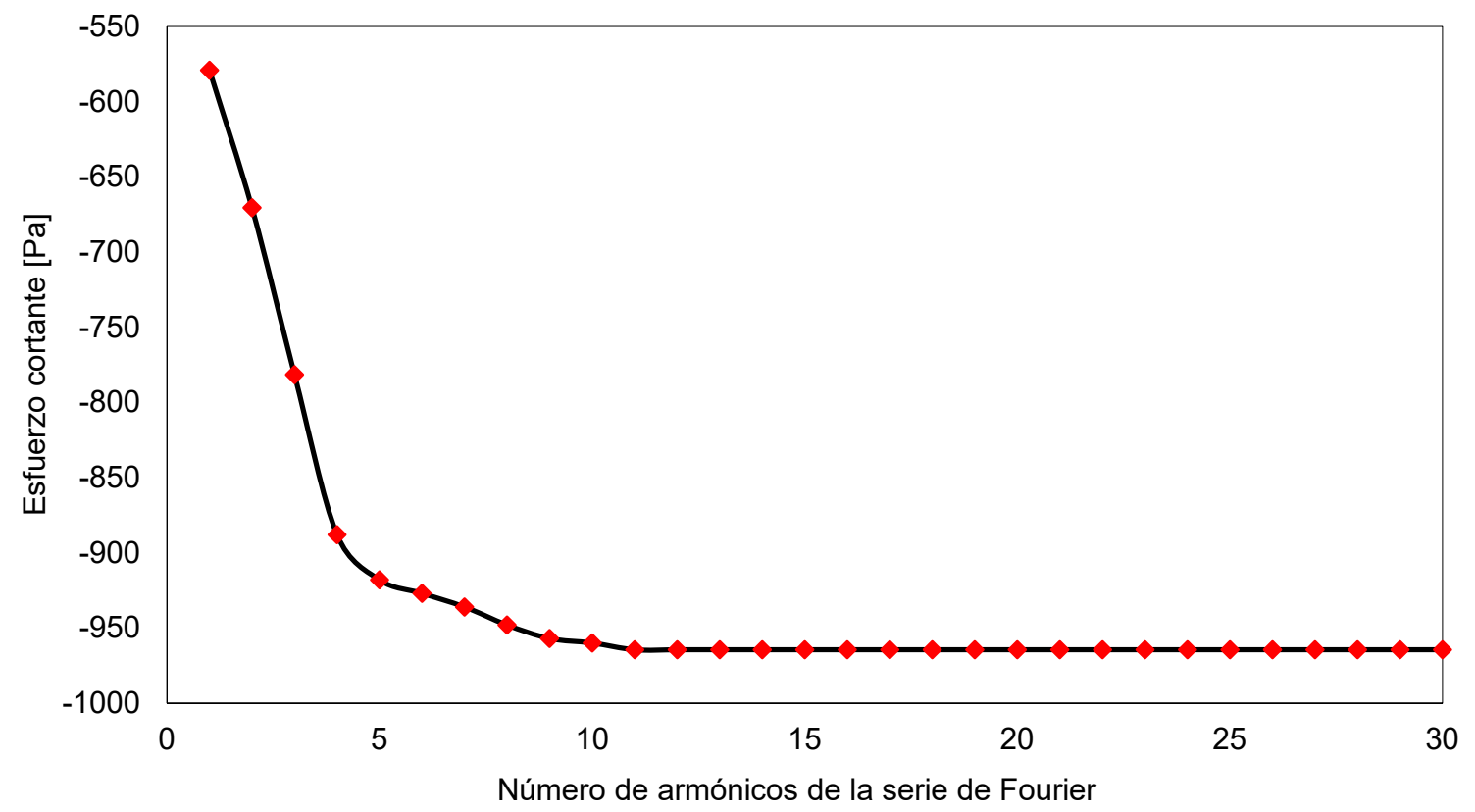

Fig. 6: Convergencia del esfuerzo cortante $\left(\tau_{\mathrm{xy}}\right)$ en el centro de la placa con el número de armónicos

\section{Resultados de la simulación numérica}

En las figuras 7 y 8 se pueden visualizar los resultados obtenidos para los esfuerzos normales y cortantes que actúan sobre la placa, dichos esfuerzos están calculados a lo largo de su línea central, es decir para a/2 (16 m) y con intervalos de b/16 de longitud es decir cada $4 \mathrm{~m}$. La figura 5 muestra la convergencia del nivel de esfuerzo normal según el número de elementos finitos presentes en la malla.

A: Statical Structural

Figure

Type: Normal Stress(X Axis) - Top/Bottom Unit: Pa

1,54601 e Max
$1,2134 \mathrm{e} 9$
$8,6674 \mathrm{e} 8$
$5,2004 \mathrm{e} 8$
$1,7335 \mathrm{e} 8$
$-1,7335 \mathrm{e} 8$
$-5,2004 \mathrm{e} 8$
$-8,6674 \mathrm{e} 8$
$-1,2134 \mathrm{e} 9$
$-1,5601 \mathrm{e} 9 \mathrm{Min}$
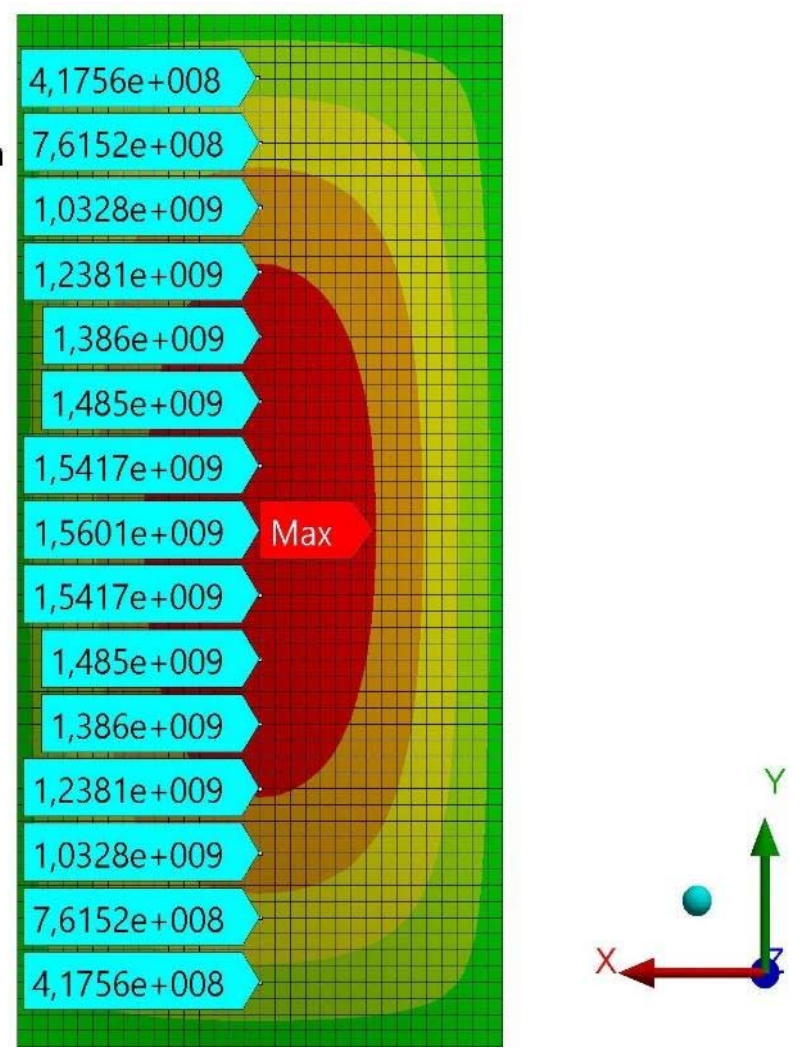

Fig. 7: Simulación en ANSYS de esfuerzos normales $\left(\sigma_{x}\right)$ inducidos en la placa rectangular plana. 

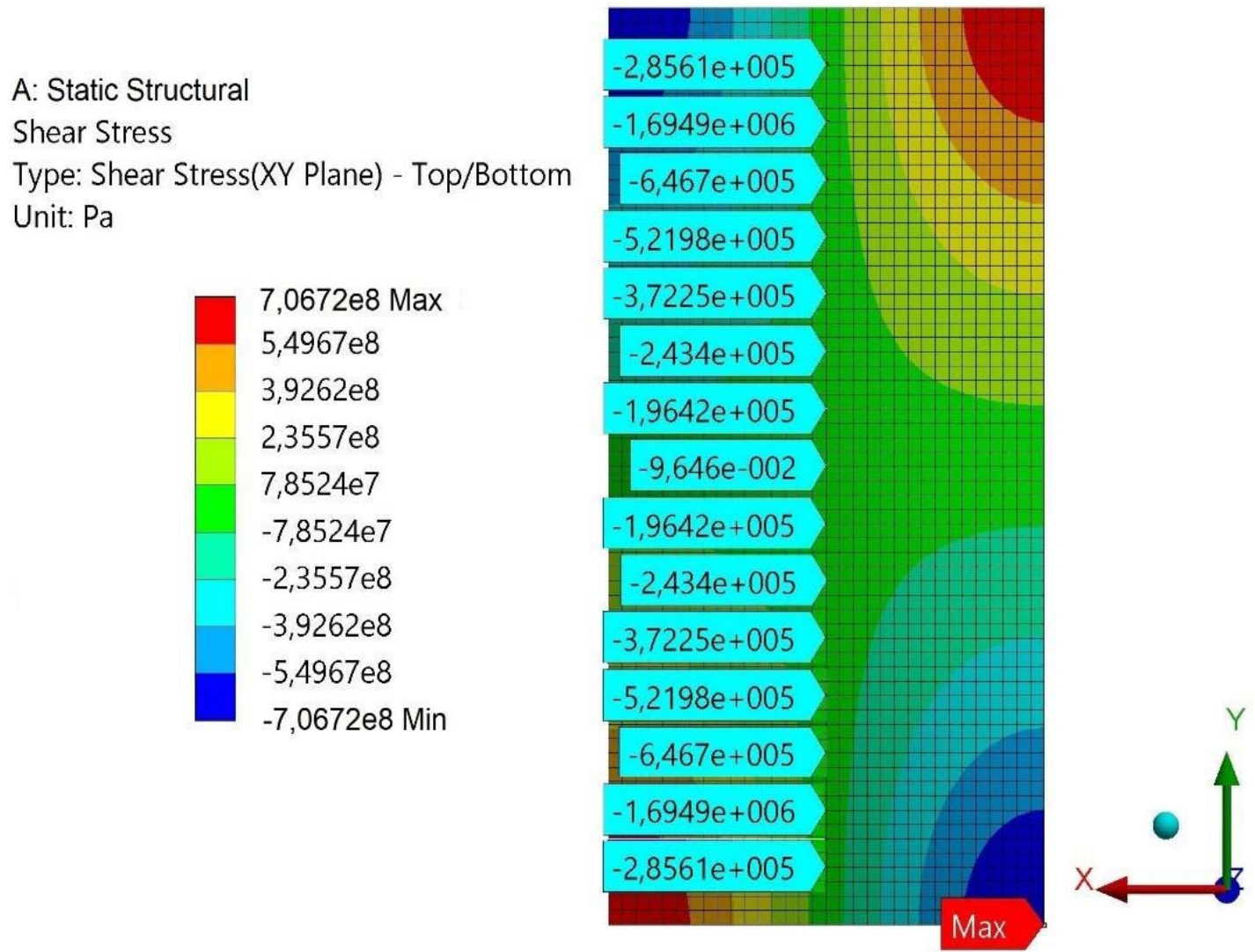

Fig. 8: Simulación en ANSYS de esfuerzos cortantes $\left(\tau_{x y}\right)$ inducidos en la placa rectangular plana

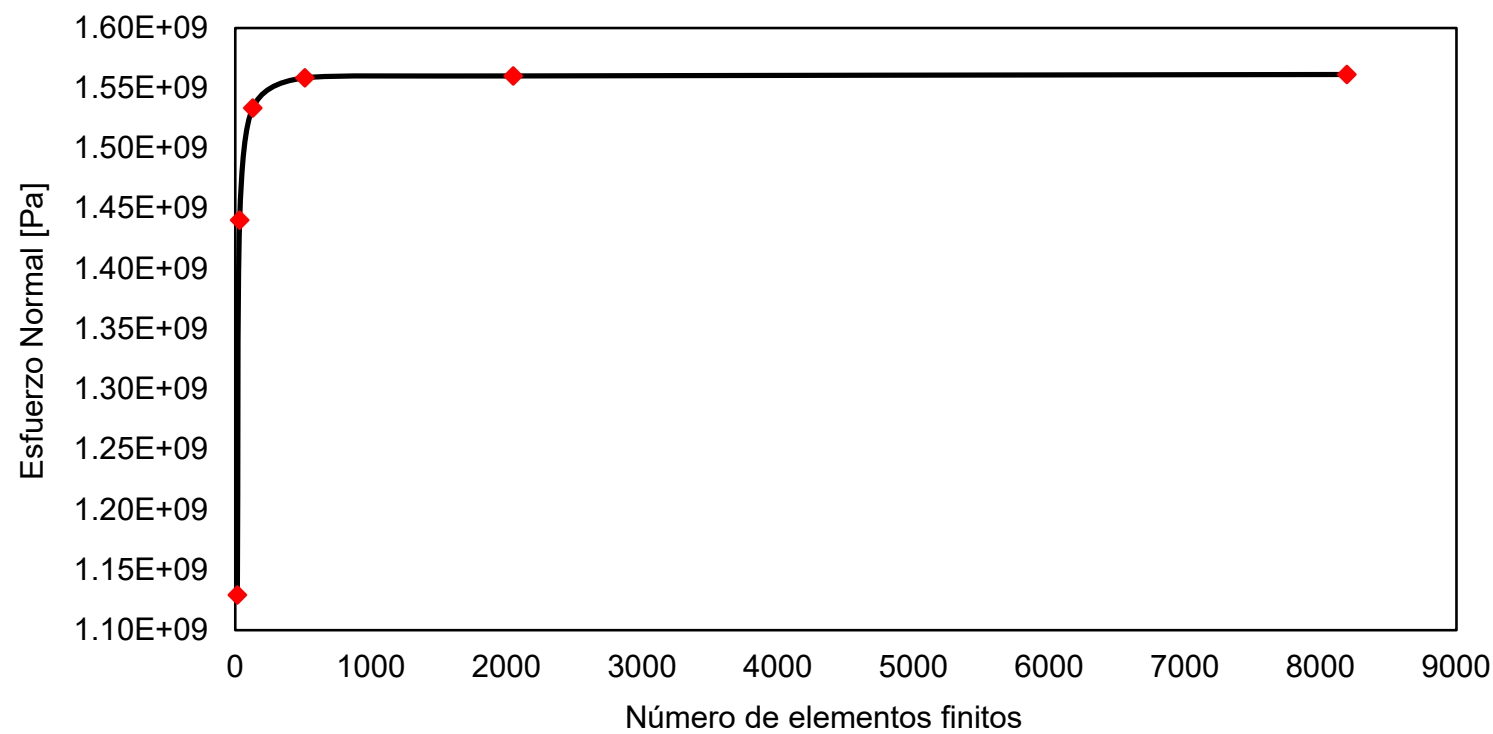

Fig. 9: Convergencia de la malla en función del número de elementos finitos para el esfuerzo normal a lo largo del eje $x$ $\left(\sigma_{x}\right)$ en el centro de la placa.

\section{Validación de la Simulación Numérica}

Para realizar la validación de los resultados se realizó una prueba t para muestras independientes con un nivel de significancia del $95 \%$ entre los valores de esfuerzo normal y cortante inducidos en la placa sometida a tres niveles de presión $10 \mathrm{~Pa}, 100 \mathrm{~Pa}$ y $1000 \mathrm{~Pa}$. Dichos valores de esfuerzo fueron obtenidos mediante el modelo de simulación numérica por elementos finitos (datos experimentales) y las soluciones analíticas (datos de control). En las tablas 3 y 4 se muestran los valores de esfuerzos normales y cortantes respectivamente para los dos tipos de soluciones, en este artículo se muestran solo los valores correspondientes a los resultados de presión de $1000 \mathrm{~Pa}$ en la placa. 
Tabla 3: Valores de esfuerzo normal $\left(\sigma_{\mathrm{x}}\right)$ analítico y por elementos finitos de la placa plana.

\begin{tabular}{|c|c|c|c|c|c|c|c|c|}
\hline $\mathrm{L}[\mathrm{m}]$ & & $\mathrm{b} / 16$ & $\mathrm{~b} / 8$ & $3 b / 16$ & $\mathrm{~b} / 4$ & $5 b / 16$ & $3 \mathrm{~b} / 8$ & $7 b / 16$ \\
\hline $\begin{array}{c}\sigma_{\mathrm{x}} \\
{[\mathrm{Pa}]}\end{array}$ & Analítico & $4,1647 \cdot 10^{8}$ & $7,6324 \cdot 10^{8}$ & $1,0422 \cdot 10^{9}$ & $1,2350 \cdot 10^{9}$ & $1,3870 \cdot 10^{9}$ & $1,4912 \cdot 10^{9}$ & $1,5365 \cdot 10^{9}$ \\
\hline $\begin{array}{c}\sigma_{x} \\
{[\mathrm{~Pa}]}\end{array}$ & $\begin{array}{l}\text { Elementos } \\
\text { finitos }\end{array}$ & $4,1756 \cdot 10^{8}$ & $7,6152 \cdot 10^{8}$ & $1,032 \cdot 10^{9}$ & $1,2381 \cdot 10^{9}$ & $1,386 \cdot 10^{9}$ & $1,485 \cdot 10^{9}$ & $1,5417 \cdot 10^{9}$ \\
\hline $\mathrm{L}[\mathrm{m}]$ & & $\mathrm{b} / 2$ & $9 b / 16$ & $5 b / 8$ & $11 \mathrm{~b} / 16$ & $3 b / 4$ & $13 b / 16$ & $7 \mathrm{~b} / 8$ \\
\hline $\begin{array}{c}\sigma_{x} \\
{[\mathrm{~Pa}]}\end{array}$ & Analítico & $1,5453 \cdot 10^{9}$ & $1,5365 \cdot 10^{9}$ & $1,4912 \cdot 10^{9}$ & $1,3870 \cdot 10^{9}$ & $1,235 \cdot 10^{9}$ & $1,0346 \cdot 10^{9}$ & $7,6324 \cdot 10^{8}$ \\
\hline $\begin{array}{c}\sigma_{\mathrm{x}} \\
{[\mathrm{Pa}]}\end{array}$ & $\begin{array}{l}\text { Elementos } \\
\text { finitos }\end{array}$ & $1,5601 \cdot 10^{9}$ & $1,5417 \cdot 10^{9}$ & $1,485 \cdot 10^{9}$ & $1,386 \cdot 10^{9}$ & $1,2381 \cdot 10^{9}$ & $1,032 \cdot 10^{9}$ & $7,6152 \cdot 10^{8}$ \\
\hline $\mathrm{L}[\mathrm{m}]$ & & $15 \mathrm{~b} / 16$ & & & & & & \\
\hline $\begin{array}{c}\sigma_{x} \\
{[\mathrm{~Pa}]}\end{array}$ & Analítico & $4,1647 \cdot 10^{8}$ & & & & & & \\
\hline $\begin{array}{c}\sigma_{\mathrm{x}} \\
{[\mathrm{Pa}]}\end{array}$ & $\begin{array}{l}\text { Elementos } \\
\text { finitos }\end{array}$ & $4,1756 \cdot 10^{8}$ & & & & & & \\
\hline
\end{tabular}

Tabla 4: Valores de esfuerzo cortante $\left(\tau_{\mathrm{xy}}\right)$ analítico y por elementos finitos de la placa plana.

\begin{tabular}{|c|c|c|c|c|c|c|c|}
\hline $\mathrm{L}[\mathrm{m}]$ & & $b / 16$ & $\mathrm{~b} / 8$ & $3 b / 16$ & $\mathrm{~b} / 4$ & $5 b / 16$ & $3 b / 8$ \\
\hline$\tau_{x y}[\mathrm{~Pa}]$ & Analítico & $-2,8903 \cdot 10^{5}$ & $-1,7203 \cdot 10^{6}$ & $-6,5446 \cdot 10^{5}$ & $-5,2824 \cdot 10^{5}$ & $-3,7708 \cdot 10^{5}$ & $-2,4656 \cdot 10^{5}$ \\
\hline $\mathrm{L}[\mathrm{m}]$ & $\begin{array}{l}\text { Elementos } \\
\text { finitos }\end{array}$ & $-2,8561 \cdot 10^{5}$ & $-1,6949 \cdot 10^{6}$ & $-6,4670 \cdot 10^{5}$ & $-5,2198 \cdot 10^{5}$ & $-3,7225 \cdot 10^{5}$ & $-2,4340 \cdot 10^{5}$ \\
\hline$\tau_{\text {xy }}[\mathrm{Pa}]$ & & $7 b / 16$ & $\mathrm{~b} / 2$ & $9 b / 16$ & $5 \mathrm{~b} / 8$ & $11 \mathrm{~b} / 16$ & $3 b / 4$ \\
\hline $\mathrm{L}[\mathrm{m}]$ & Analítico & $-1,9877 \cdot 10^{5}$ & $-9,7713 \cdot 10^{2}$ & $-1,9877 \cdot 10^{5}$ & $-2,4656 \cdot 10^{5}$ & $-3,7708 \cdot 10^{5}$ & $-5,2824 \cdot 10^{5}$ \\
\hline$\tau_{\mathrm{xy}}[\mathrm{Pa}]$ & $\begin{array}{l}\text { Elementos } \\
\text { finitos }\end{array}$ & $-1,9642 \cdot 10^{5}$ & $-9,6465 \cdot 10^{2}$ & $-1,9642 \cdot 10^{5}$ & $-2,4340 \cdot 10^{5}$ & $-3,7225 \cdot 10^{5}$ & $-5,2198 \cdot 10^{5}$ \\
\hline$L[\mathrm{~m}]$ & & $13 b / 16$ & $7 \mathrm{~b} / 8$ & $15 b / 16$ & & & \\
\hline$\tau_{\mathrm{xy}}[\mathrm{Pa}]$ & Analítico & $-6,5446 \cdot 10^{5}$ & $-1,7169 \cdot 10^{6}$ & $-2,8903 \cdot 10^{5}$ & & & \\
\hline $\mathrm{L}[\mathrm{m}]$ & $\begin{array}{l}\text { Elementos } \\
\text { finitos }\end{array}$ & $-6,4670 \cdot 10^{5}$ & $-1,6949 \cdot 10^{6}$ & $-2,8561 \cdot 10^{5}$ & & & \\
\hline
\end{tabular}

El valor del estadístico de prueba t para muestras independientes es igual a 086 que es mayor al nivel de significancia de 0,05 por lo tanto se acepta la hipótesis nula $\mathrm{H}_{0}$, es decir que no hay diferencia significativa entre en las medias de los valores de esfuerzo normal y cortante entre las soluciones analíticas y las del modelo de simulación, por lo tanto, dicho modelo se valida.

\section{CONCLUSIONES}

A partir del estudio presentado se puede concluir: 1) Los modelos de simulación numérica por elementos finitos que utilizan el software ANSYS 19.1 son una solución extremadamente versátil y eficiente para el desarrollo de proyectos de ingeniería y en este caso en particular para la determinación de esfuerzos normales y cortantes en placas planas sometidas a cargas uniformente distribuidas. 2) Los resultados del análisis de esfuerzos normales y cortantes para placas planas rectangulares isotrópicas sometidas a presión uniformemente distribuida muestran que no hay diferencias estadísticas significativas entre las soluciones obtenidas por el método de elementos finitos y las soluciones analíticas. 3) Para obtener resultados satisfactorios en la simulación del modelo, se encontró que el valor óptimo para la convergencia correspondió en éste caso a 2048 elementos finitos. 4) Los modelos de simulación numérica se deben utilizar como método complementario a los métodos analítico y experimental, con varias ventajas en el desarrollo de proyectos de ingeniería, de tal forma que les permitan a los ingenieros tener más recursos para lograr un buen balance en la calidad, el tiempo y el costo de sus productos, equipos y procesos. 


\section{REFERENCIAS}

Alfonso, I., Rodríguez-Iglesias, V., y Figueroa, I.A., Potencialidades computacionales del método de los elementos finitos para la modelación y simulación de materiales compuestos: Revisión, Revista Materia, 20(2), 293-303 (2015)

Birman, V., Plate structures, $1^{\circ}$ Ed.,53-57, Springer, Londres, Inglaterra (2011)

Cronin, P.J., Plane stress FEA problem which students can solve using an inexpensive calculator, American Society for Engineering Education Annual Conference \& Exposition, USA (2001)

Del Rey, R., Alba, J., Ramis, J., y Escuder, E., Aplicación del método de los elementos finitos para la simulación de las transmisiones por flanco en uniones con suelos flotantes, https://dx.doi.org/10.4067/S0718-07642010000600009, Información Tecnológica, 21(6), 67-78 (2010)

Gurunath, V.S., Fundamental concept of finite element method, https://doi.org/10.13140/RG.2.1.2914.1604, (India) DACOE (2015)

Hassan, A., Kurgan, N., Modeling and buckling analysis of rectangular plates in ANSYS,

https://doi.org/10.24107/ijeas.531011, International Journal of Engineering and Applied Sciences,11 (1),310-329 (2019)

Herrera, D., Desarrollo de una herramienta numérica de análisis para losas de hormigón armado sometidas a aceleraciones verticales sísmicas.,https://dx.doi.org/10.4067/S0718-28132011000200001, Obras y Proyectos, (10), 414, (2011)

Li, X.F., y Hu, Z.L., Generalization of plane stress and plane strain states to elastic plates of finite thickness, https://doi.org/10.1007/s10659-020-09768-7, Journal of Elasticity,140, 243-256 (2020)

Manjunath, G.A., Athresh, K.R., Arvind, B., y Punith, R., Finite element modelling for stress analysis of a rhombic skew plate structure, International Journal of Engineering Research \& Technology (IJERT), (2), 2378-2387 (2014)

Mohtaram, F., Kahnamouei, J.T., Shariati, M.B., y Behjat, B., Experimental and numerical Investigation of buckling in rectangular steel plates with groove-shaped cutouts, https://doi.org/10.1631/jzus.A1100226, Journal of Zhejiang University-Science a (Applied Physics \& Engineering), 13(6), 469-480 (2012)

Moreno, J., Ocampo, J., y Giraldo, L., Simulación de una celda calorimétrica mediante elementos finitos, https://dx.doi.org/10.4067/S0718-07642007000200004, Información Tecnológica, 18(2), 25-35 (2007)

Muhammad, A., Ali, M. A., y Shanono, I.H., Finite element analysis of a connecting rod in ANSYS, https://doi.org/10.1088/1757-899X/736/2/022119, Materials Science and Engineering, 736 (2020)

Pallares, M., y Rodríguez, C.W., Modelación del fenómeno de vibración forzada formulando una analogía eléctrica con el programa de elementos finitos "Ansys", https://doi.org/10.15446/inginvestig.v29n1.15137, Revista Ingeniería e Investigación, 29(1), 5-12 (2009)

Ramírez, J., L., y Roldán, L., F., Nueva era del diseño en ingeniería, Revista Facultad De Ingeniería Universidad De Antioquia, (14), 146-156 (2016)

Roylance, D., Finite element analysis, Department of Materials Science and Engineering Massachusetts (2001)

Sadrnejad, S. A., Saedi, D., y Ziaei, M., Vibration equations of thick rectangular plates using mindlin plate theory, Journal of Computer Science,5 (11), 838-842 (2009)

Timoshenko, S.P., y Gere, J.M., Theory of elastic stability, $2^{\circ}$ Ed.,319-325, McGraw-Hill, New York, USA (2009)

Ugural, A.C., Stresses in beams, plates, and shells, $3^{\circ}$ Ed.,175-176, Taylor \& Francis Group, Boca Raton, USA (2010)

Vanam, B., Rajyalakshmi M., y Inala, R., Static analysis of an isotropic rectangular plate using finite element analysis (FEA), https://doi.org/10.5897/JMER11.088, Journal of Mechanical Engineering Research, 4(4), 148-162 (2012)

Vélez, W. D., Thomson, P., Ajuste de modelos de elementos finitos, Dyna, 76(158),177-189 (2009)

Ventsel, E., y Krauthammer, T., Thin plates and shells: theory: analysis, and applications, $1^{\circ}$ Ed., CRC Press, New York, USA (2001)

Yuwaraj, M.G., y Param, D.G., Bending analysis of thick isotropic plates by using 5 th order shear deformation theory, https://doi.org/10.22055/JACM.2016.12366, Journal of Applied and Computational Mechanics, 2(2), 80-95 (2016) 
